\title{
An Integrated Nomogram Combining Clinical Factors and Microtubule-Associated Protein 1 Light Chain 3B Expression to Predict Postoperative Prognosis in Patients with Intrahepatic Cholangiocarcinoma
}

\author{
Liang Chen, $\mathrm{MD}^{1,2}$ \\ Hongyuan $\mathrm{Fu}, \mathrm{MD}^{13}$ \\ Tongyu Lu, MD1,2 \\ Jianye Cai, MD1,2 \\ Wei Liu, PhD2 \\ Jia Yao, MD',2 \\ Jinliang Liang, $\mathrm{MS}^{2}$ \\ Hui Zhao, MD',2 \\ Jiebin Zhang, $M D^{1,2}$ \\ Jun Zheng, MD1,2 \\ Yingcai Zhang, MD ${ }^{1,2}$ \\ Yang Yang, MD1,2
}

*A list of author's affiliations appears at the end of the paper.

Correspondence: Yingcai Zhang, MD Department of Hepatic Surgery and Liver Transplantation Center, Third Affiliated Hospital of Sun Yat-sen University,

Guangzhou 510630, China

Tel: 86-20-85252177

Fax: 86-20-85252276

E-mail: zhangyc3@mail.sysu.edu.cn

Co-correspondence: Jun Zheng, MD Department of Hepatic Surgery and Liver Transplantation Center, Third Affiliated

Hospital of Sun Yat-sen University,

Guangzhou 510630, China

Tel: 86-20-85252177

Fax: 86-20-85252276

E-mail: zhengj67@mail2.sysu.edu.cn

Co-correspondence: Yang Yang, MD

Department of Hepatic Surgery and Liver

Transplantation Center, Third Affiliated

Hospital of Sun Yat-sen University,

Guangzhou 510630, China

Tel: 86-20-85252177

Fax: 86-20-85252276

E-mail: yysysu@163.com

Received July 22, 2019

Accepted September 30, 2019

Published Online October 7, 2019

*Liang Chen, Hongyuan Fu, Tongyu Lu, and Jianye Cai contributed equally to this work.

\section{Purpose}

Microtubule-associated protein 1 light chain 3B (LC3B) serves as a key component of autophagy, which is associated with the progression of carcinoma. Yet, it is still unclear whether LC3B is also an independent risk factor for intrahepatic cholangiocarcinoma (ICC). We aim to explore the predictive value of LC3B on prognosis of ICC, and to establish a novel and available nomogram to predict relapse-free survival (RFS) and overall survival (OS) for these patients after curative-intent hepatectomy.

\section{Materials and Methods}

From August 2004 to March 2017, 105 ICC patients were eligibly enrolled in the Third Affiliated Hospital of Sun Yat-sen University. Preoperative clinical information of enrolled patients was collected. Expression LC3B in the ICC specimen was detected by immunohistochemistry.

\section{Results}

The 5-year RFS and OS in this cohort were $15.7 \%$ and $29.6 \%$, respectively. On multivariate Cox regression analysis, independent risk factors for 5-year OS were cancer antigen 125 , microvascular invasion, LC3B expression and lymph node metastasis. Except for the above 4 factors, neutrophil/lymphocyte ratio and tumor differentiation were independent factors for 5-year RFS. The area under the curve of nomograms for OS and RFS were 0.820 and 0.747 , respectively.

\section{Conclusion}

The nomograms based on LC3B can be considered as effective models to predict postoperative survival for ICC patients.

Key words

Intrahepatic cholangiocarcinoma, Autophagy, LC3B, Nomogram, Prognosis 


\section{Introduction}

Intrahepatic cholangiocarcinoma (ICC) is the second common primary hepatic malignancy which accounts for $10 \%$ $20 \%$ and arises from the endothelial cells of intrahepatic bile duct $[1,2]$. Although a reported incidence of ICC remained rare with $0.95 / 100,000$ in the United State and 0.5-3.4/ 100,000 in Western Europe, the morbidity has been increasing over the past decade [3]. To date, surgical resection is still the mainstay of curative approach for ICC $[4,5]$. Unfortunately, prognosis of ICC is poor due to a high incidence of local relapse and/or distant metastasis after liver resection [6-8]. Therefore, the accurate prediction of tumor biologic behavior after surgery is crucial for guiding individualized post-operative therapy and improving ICC survival outcomes.

Autophagy, an intracellular catabolic mechanism, is an important biological process for maintaining cellular homeostasis by trapping and further degrading the long-lived organelles and proteins [9]. Previous studies showed that autophagy was liked a two-edged sword that was beneficial to cell survival with its ability to suppress apoptosis, while led to the initiation and development of diseases, including neurodegenerative diseases and autoimmune disorders $[10,11]$. In response to chemotherapy, hypoxia and nutrient deprivation, tumor cells activate autophagy, which maintains cell survival as well as strengthens their ability of metastasis and therapeutic resistance [12,13]. Increasing number of researches have demonstrated the correlation between autophagic activity and tumor characteristics and detected the autophagy-related proteins expression in tumor specimen to analyze patients' clinical outcomes. Microtubule-associated protein 1 light chain 3B (LC3B) is an important protein of the autophagic process which participates in the formation of autophagosomal membranes. LC3B is a marker for evaluating functional basal autophagy and is believed to be upregulated in several cancer types $[14,15]$. Other studies have shown that high LC3B expression was significantly associated with poor survival outcomes of patients with gastric cancer, breast cancer and colorectal cancer $[15,16]$. Mechanically, LC3B may play an important role in maintaining the stemness of cancer cells [17]. Tumor progression and metastasis is regulated by LC3B-mediated macrophagy $[18,19]$. However, it is still unclear that whether LC3B plays a predictive role for early metastasis and postoperative survival of ICC patients.

Nomogram is a statistical model specifically used for individualized prediction of clinical decision making with quantization and incorporation of independent risk variables. Nomogram has also been widely developed to individually prediction accuracy of various cancer types, and presented more advantage over the traditional staging systems. Recently, both Wang et al. [20] and Hyder et al. [21] reported accurate nomograms to predict long-term prognostic outcomes after hepatectomy for ICC with large sample size from a single center and multicenter, respectively. However, these nomograms were constructed only integrating clinicopathologic variables other than significant biomarkers. In this study, we analyzed LC3B expression in ICC specimens by immunohistochemical (IHC) analysis and detected its correlation with relapse-free survival (RFS) and overall survival (OS) of ICC patients after surgery. In addition, we screened the independent predictors of clinical information and established nomograms integrating clinicopathologic variables and LC3B expression. To compare its efficacy of prognostic prediction, we aim to develop highly sensitive models which based on specific pathologic biomarkers to predict RFS and OS of ICC patients who recovered from a curative intended resection.

\section{Materials and Methods}

\section{Patients and tissue specimens}

In this study, we collected ICC specimen from 105 consecutive patients with ICC who underwent curative intended hepatectomy from August 2004 to March 2017 at the Third Affiliated Hospital of Sun Yat-sen University (Guangzhou, China). All tumor specimens were histologically confirmed as ICC by a pathologist. Staging was determined following the seventh edition of the American Joint Committee on Cancer (AJCC) staging of ICC. Detailed information, including demographic, clinic and imagology data as well as neutrophil, lymphocyte, monocyte, platelet count, and serum cancer antigen 125 (CA-125), from enrolled patients before surgery were obtained from handwritten and electronic medical records. The patients were excluded, which suffered from acute condition, including viral or bacterial infections, massive hemorrhage and a histology of taking hematopoietic drugs before surgery. Neutrophil/lymphocyte ratio (NLR) was calculated as (absolute neutrophil count) / (absolute lymphocyte count).

\section{Follow-up}

After operation, the enrolled patients regularly received follow-up in the outpatient of the Third Affiliated Hospital of Sun Yat-sen University once every 3 months in the first two years and then every 6 months thereafter. A detail histology and a physical examination were routinely carried out 
at each of the follow-up appointments. The levels of serum $\alpha$-fetoprotein, CA-125, and carcinoembryonic antigen (CEA) and liver function tests in peripheral venous blood were detected, and abdominal ultrasound was performed. Magnetic resonance imaging or plain/ enhanced abdominal computed tomography (CT) scans was carried out once every 6 months. In addition, enhanced CT/positron emission tomography CT scan for lung or bone scan was carried out immediately when distant metastasis was suspected. RFS and OS were considered as the primary endpoints. RFS was calculated as the interval period between surgery and last tumor investigation/last follow-up/relapse/death, while OS was defined as the date of liver resection to the time of death or last follow-up.

\section{Immunohistochemistry and quantification of LC3B expression}

Four-micrometer-thick paraffin-embedded ICC sections were made and stained with hematoxylin and eosin for confirming specimen integrity. To perform immunohistochemistry, the sections were deparaffinized, rehydrated and repaired by ethylenediaminetetraacetic acid ( $\mathrm{pH}$ 8.0). The peroxidase activity in tissues was quenched using $3 \% \mathrm{H}_{2} \mathrm{O}_{2}$. The sections were incubated with bovine serum albumin to block nonspecific antibody for 30 minutes. The primary antibody (LC3B, 1:200, Abcam, Cambridge, MA) was used to treat the sections at $4^{\circ} \mathrm{C}$ overnight. After washed twice with phosphate buffer saline and incubated with secondary antibody (Dako, Carpinteria, CA) for 30 minutes at $37^{\circ} \mathrm{C}$, the samples were observed under a light microscope (Leica, Wetzlar, Germany) by treating with diaminobenzidine.

Immunostaining analysis was performed by two independent observers (L. Chen and H. Fu) according to previous study [22]. Sections were graded according to percent staining (score of 0 to 4 points: 0 points, $0 \%$; 1 points, $1 \%-5 \%$; 2 points, $6 \%-10 \% ; 3$ points, $11 \%-50 \%$; 4 points, $>50 \%$ ) and intensity (scale of 0 to 3 points: 0 points, none; 1 points, weak; 2 points, moderate; 3 points, marked). The immunochemical score was calculated as the product of staining and intensity, which was similar to the immune-reactive score from Remmele and Stegner [23]. For further analysis, the IHC score were stratified into "low" or "high" expression based on the optimal cut-off value with best sensitivity and specificity.

\section{Statistical analysis}

We used SPSS ver. 20.0 (IBM Corp., Armonk, NY) and R software ver. 3.4.4 (Institute for Statistics and Mathematics, Vienna, VIC, Austria) to conduct the following analysis. The continuous variables were expressed as median (interquartile range) and compared by Students t test or the Mann-
Whitney $\mathrm{U}$ test for variables with an abnormal distribution. The categorical variables were compared by chi-square test. Survival analysis was performed using Kaplan-Meier method and log-rank method was used to test the differences between groups. Univariable and multivariable Cox proportional hazard regression models were used to analyze the OS and RFS of patients with ICC, in which hazards ratio and 95\% confidence interval (CI) were shown.

Nomogram for final prognostic factors associated with 1-, 3-, and 5-year survival was established. The performance of the nomograms was evaluated by calibration curve and area under the curve (AUC), and were evaluated by comparing Kaplan-Meier estimates predicted by nomogram and observed survival probabilities, and 1,000-resampled bootstrap is applied to these activities. The optimal cut-off points of the NLR and total points of nomogram were determined by using the X-tile software ver. 3.6.1 (Yale University, New Haven, CT). All the tests were two sides and $\mathrm{p}<0.05$ were considered to be statistically significant.

\section{Ethical statement}

The study was approved by the Clinical Research Ethic Committee of the Third Affiliated Hospital of Sun Yat-sen University (2017-065-01), and all patients signed the informed consent to participate in this study.

\section{Results}

\section{Patients' baseline characteristics}

The baseline characteristics of enrolled patients are listed in Table 1. Data from 105 patients with ICC who met the eligible criteria were included in this study. Patients' average age was 57.0 years (range, 49.0 to 64.0 years). Mean tumor diameter was $5.3 \mathrm{~cm}$. Patients with hepatitis B virus infection accounted for $40 \%$. Based on the histological features, 25 patients $(23.8 \%)$ had lymph node metastasis (LNM), 33 (31.4\%) had vein invasion, $20(19.0 \%)$ had adjacent tissue infiltration. And the mean RFS time was 8.0 months (range, 4.0 to 20.0 months). The 1-, 3-, and 5-year RFS rates were 43.3\%, 23.9\% and $15.7 \%$, respectively. The median OS time was 15.0 months (range, 8.0 to 36.5 months). The 1-, 3-, and 5-year OS rates were $62.6 \%, 37.2 \%$ and $29.6 \%$, respectively. Positive expression of LC3B was mainly located in the neoplastic parenchyma (Fig. 1). The patients were stratified into low (79 patients) and high (26 patients) LC3B expression (Table 2). Kaplan-Meier survival curves were performed and it was indicated that high level of LC3B expression in tumor sec- 
Table 1. Baseline characteristics of ICC patients

\begin{tabular}{|c|c|}
\hline Variable & Value $(n=105)$ \\
\hline \multicolumn{2}{|l|}{ Sex } \\
\hline Male & $60(57.1)$ \\
\hline Female & $45(42.9)$ \\
\hline Age (yr) & $57.0(49.0-64.0)$ \\
\hline \multicolumn{2}{|l|}{ Hepatitis virus infection } \\
\hline HBV & $42(40.0)$ \\
\hline $\mathrm{HCV}$ & $2(1.9)$ \\
\hline \multicolumn{2}{|l|}{ Peripheral blood cells $\left(\times 10^{9} / \mathrm{L}\right)$} \\
\hline Neutrophils $\left(\times 10^{9} / \mathrm{L}\right)$ & $4.17(3.23-5.48)$ \\
\hline Lymphocytes $\left(\times 10^{9} / \mathrm{L}\right)$ & $1.75(1.47-2.10)$ \\
\hline NLR & $2.34(1.68-3.59)$ \\
\hline $\operatorname{ALT}(\mathrm{U} / \mathrm{L})$ & $25.0(17.0-42.5)$ \\
\hline AST (U/L) & $28.0(22.0-41.0)$ \\
\hline GGT (U/L) & $64.0(34.5-113.0)$ \\
\hline Albumin $(g / L)$ & $38.9(36.0-41.8)$ \\
\hline TBIL $(\mu \mathrm{mol} / \mathrm{L})$ & $12.8(10.3-19.5)$ \\
\hline \multicolumn{2}{|l|}{ Serum tumor markers } \\
\hline CA-199 (U/mL) & $50.99(20.53-152.35)$ \\
\hline CEA $(\mathrm{g} / \mathrm{L})$ & $2.16(1.40-3.74)$ \\
\hline CA-125 (U/mL) & $20.02(14.28-36.19)$ \\
\hline $\operatorname{AFP}(\mathrm{ng} / \mathrm{mL})$ & $3.56(2.52-7.04)$ \\
\hline Diameter (cm) & $5.3(4.2-7.5)$ \\
\hline \multicolumn{2}{|l|}{ Tumor number } \\
\hline 1 & $78(74.3)$ \\
\hline 2 & $6(5.7)$ \\
\hline 3 & $1(1.0)$ \\
\hline$>3$ & $20(19.0)$ \\
\hline \multicolumn{2}{|l|}{ Tumor location } \\
\hline Left hemi liver & $47(44.8)$ \\
\hline Right hemi liver & $49(46.7)$ \\
\hline Left and right hemi liver & $9(8.6)$ \\
\hline \multicolumn{2}{|l|}{ Hepatolithiasis } \\
\hline Positive & $21(20.0)$ \\
\hline Negative & $84(80.0)$ \\
\hline \multicolumn{2}{|l|}{ Vein invasion } \\
\hline Positive & $33(31.4)$ \\
\hline Negative & $72(68.6)$ \\
\hline \multicolumn{2}{|l|}{ TNM (AJCC, 2010) } \\
\hline I & $33(31.4)$ \\
\hline II & $31(29.5)$ \\
\hline III & $26(24.8)$ \\
\hline IV & $15(14.3)$ \\
\hline \multicolumn{2}{|l|}{ Differentiation } \\
\hline Well & $12(11.4)$ \\
\hline Moderate & $62(59.0)$ \\
\hline Poor & $31(29.5)$ \\
\hline \multicolumn{2}{|l|}{ Surgical margin } \\
\hline Positive & $15(14.3)$ \\
\hline Negative & $90(85.7)$ \\
\hline
\end{tabular}

(Continued)
Table 1. Continued

\begin{tabular}{lc} 
Variable & Value (n=105) \\
MVI & \\
Positive & $27(25.7)$ \\
Negative & $78(74.3)$ \\
LNM & \\
$\quad$ Positive & $25(23.8)$ \\
Negative & $80(76.2)$ \\
Adjacent tissue infiltrated & \\
$\quad$ Positive & $20(19.0)$ \\
$\quad$ Negative & $85(81.0)$ \\
OS (\%) & \\
1-Year & 62.6 \\
3-Year & 37.2 \\
5-Year & 29.6 \\
RFS (\%) & \\
1-Year & 43.3 \\
3-Year & 23.9 \\
5-Year & 15.7 \\
\hline
\end{tabular}

Values are presented as number (\%) or median (range) unless otherwise indicated. ICC, intrahepatic cholangiocarcinoma; $\mathrm{HBV}$, hepatitis $B$ virus; $\mathrm{HCV}$, hepatitis $\mathrm{C}$ virus; NLR, neutrophil-to-lymphocyte ratio; AST, aspartate aminotransferase; ALT, alanine aminotransferase; GGT, gamma glutamyl transpeptidase; TBIL, total bilirubin; CEA, carcinoembryonic antigen; CA-125, cancer antigen 125; AFP, $\alpha$-fetoprotein; AJCC, American Joint Committee on Cancer; MVI, microvascular invasion; LNM, lymph node metastasis; OS, overall survival; RFS, relapse-free survival.

tions associated with poorer OS (log-rank, $\mathrm{p}=0.001$ ) and earlier relapse (log-rank, $\mathrm{p}<0.001$ ) (Fig. 1).

\section{Prognostic factors for OS and RFS}

Univariate analyses were performed to investigate the predictive role of LC3B expression in patients' survival after surgery (Tables 3 and 4). It is showed that high LC3B expression was an independent prognostic biomarker for both OS (hazard ratio [HR], 2.33; 95\% CI, 1.41 to 3.86; $\mathrm{p}=0.001$ ) and RFS (HR, 2.70; 95\% CI, 1.65 to $4.43 ; \mathrm{p}<0.001)$. Other significant predictive factors for OS include CEA (HR, 2.32; 95\% CI, 1.35 to 3.99; $\mathrm{p}=0.002$ ), $\mathrm{CA}-125$ (HR, 3.80; 95\% CI, 2.24 to 6.43; $\mathrm{p}<$ 0.001 ), NLR (HR, 2.69; 95\% CI, 1.62 to $4.46 ; \mathrm{p}<0.001$ ), tumor size (HR, 2.27; 95\% CI, 1.31 to 3.95; $\mathrm{p}=0.004)$, tumor-nodemetastasis (TNM) stage (HR, 2.16; 95\% CI 1.33 to 3.51; $\mathrm{p}=0.002)$, the number of tumor nodules (HR, $1.39 ; 95 \% \mathrm{CI}$, 1.06 to $1.82 ; \mathrm{p}=0.018$ ), LNM (HR, 2.32; 95\% CI 1.36 to 3.96; $\mathrm{p}=0.002)$, surgical margin $(\mathrm{HR}, 2.40 ; 95 \% \mathrm{CI}, 1.29$ to 4.44 ; 
Table 2. The expression level of LC3B in ICC tumor tissues $(n=105)$

\begin{tabular}{llllll}
\multirow{2}{*}{ Antigen } & \multicolumn{2}{c}{ Low expressed } & & \multicolumn{2}{c}{ High expressed } \\
\cline { 2 - 3 } \cline { 5 - 6 } & Negative & Weak & & Moderate & Strong \\
\hline LC3B & $16(15.2)$ & $63(60.0)$ & & $21(20.0)$ & $5(4.8)$ \\
\hline
\end{tabular}

Values are presented as numbers of patients $(\%)$. LC3B, microtubule-associated protein 1 light chain 3B; ICC, intrahepatic cholangiocarcinoma.

A

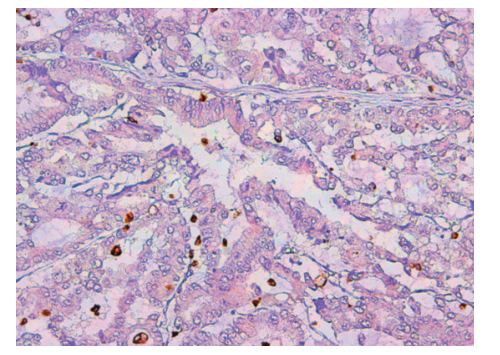

C

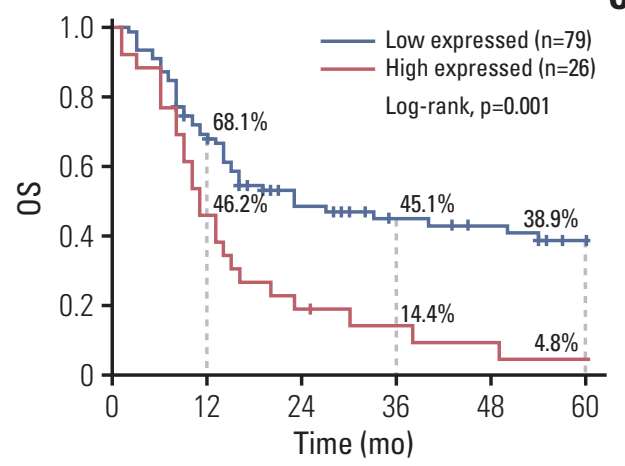

B

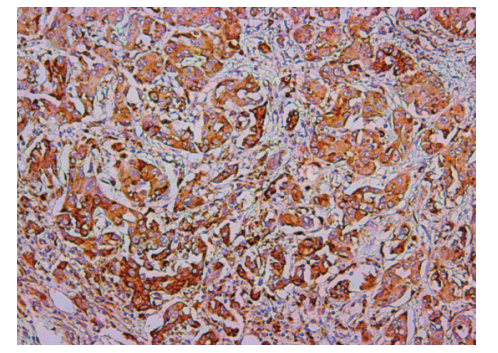

D

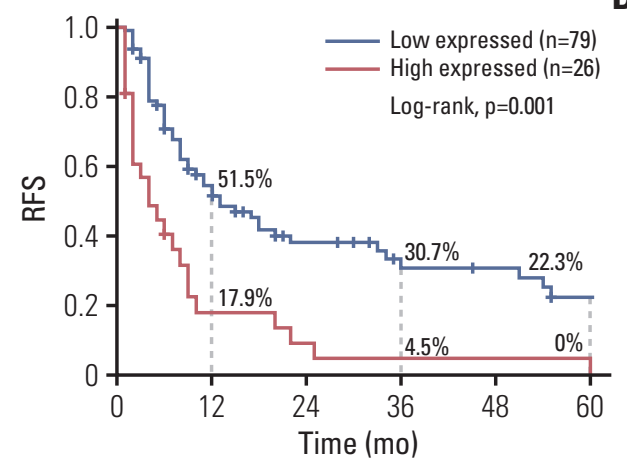

Fig. 1. Representative immunohistochemistry samples $(\times 200)$ of low (A) and high (B) microtubule-associated protein 1 light chain 3B (LC3B) expression in intrahepatic cholangiocarcinoma patients and Kaplan-Meier analysis of overall survival (OS) (C) and relapse-free survival (RFS) (D) for patients with low and high expressed LC3B after surgery.

$\mathrm{p}=0.005)$, and microvascular invasion (MVI) (HR, 2.82; 95\% CI, 1.67 to $4.74 ; \mathrm{p}<0.001)$ were independent risk factors. Besides, other significant predictive factors for RFS include CEA (HR, 1.78; 95\% CI, 1.00 to 3.17; $\mathrm{p}=0.048$ ), CA-125 (HR, $2.91 ; 95 \% \mathrm{CI}, 1.63$ to $5.19 ; \mathrm{p}<0.001)$, NLR (HR, 2.83; 95\% CI, 1.75 to $4.6 ; \mathrm{p}<0.001)$, tumor size (HR, 2.70; 95\% CI, 1.65 to $4.43 ; \mathrm{p}=0.007)$, TNM stage (HR, 2.40; $95 \% \mathrm{CI}, 1.48$ to $3.89 ; \mathrm{p}<$ $0.001)$, the number of tumor nodules (HR, 1.70; 95\% CI, 1.31 to $2.21 ; \mathrm{p}<0.001)$, LNM (HR, 2.66; 95\% CI, 1.53 to 4.63 ; $\mathrm{p}=0.001$ ), tumor differentiation (HR, $1.87 ; 95 \% \mathrm{CI}, 1.17$ to 2.98; $\mathrm{p}=0.009)$, and MVI (HR, 2.88; 95\% CI, 1.7 to 4.88; $\mathrm{p}<0.001)$.

Next, multivariate regression analyses indicated that a high expression of LC3B was also an unfavorable predictor of RFS (HR, 3.43; 95\% CI, 1.87 to 6.29; $<<0.001)$ and OS (HR,
2.16; $95 \% \mathrm{CI}, 1.22$ to 3.84; $\mathrm{p}=0.009)$. Other independent predictors for RFS and OS includes CA-125, LNM, and MVI. However, NLR (HR, 1.87; 95\% CI, 1.09 to 3.18; $\mathrm{p}=0.022)$ and tumor differentiation (HR, 1.82; 95\% CI, 1.10 to 3.02; $\mathrm{p}=0.019)$ were statistically significant for RFS but not OS.

\section{Validation of predictive ability of the nomogram for OS and RFS}

To provide the surgeon with a quantitative approach to predict ICC patients' probability of 1-, 3-, and 5-year OS, we developed a nomogram which incorporated four independent prognostic factors (CA-125, LNM, MVI, and LC3B expression). Meanwhile, according to the results of Cox regression 
Table 3. Univariate and multivariate analyses for OS in ICC patients $(n=105)$

\begin{tabular}{|c|c|c|c|c|c|c|}
\hline \multirow{2}{*}{ Variable } & \multicolumn{3}{|c|}{ Univariate analysis } & \multicolumn{3}{|c|}{ Multivariate analysis } \\
\hline & HR & $95 \%$ CI & p-value & HR & $95 \%$ CI & p-value \\
\hline Sex & 0.73 & $0.45-1.18$ & 0.200 & - & - & - \\
\hline Age $(>60$ yr vs. $<60 \mathrm{yr})$ & 0.79 & $0.48-1.29$ & 0.343 & - & - & - \\
\hline $\mathrm{HBV}$ & 0.66 & $0.4-1.1$ & 0.110 & - & - & - \\
\hline CEA $(>5.0 \mu \mathrm{g} / \mathrm{L}$ vs. $<5.0 \mu \mathrm{g} / \mathrm{L})$ & 2.32 & $1.35-3.99$ & 0.002 & - & - & 0.426 \\
\hline CA-199 (> $35 \mathrm{U} / \mathrm{mL}$ vs. $<35 \mathrm{U} / \mathrm{mL}$ ) & 1.08 & $0.65-1.79$ & 0.762 & - & - & - \\
\hline CA-125 (>35 U/mL vs. $<35 \mathrm{U} / \mathrm{mL})$ & 3.80 & 2.24-6.43 & $<0.001$ & 3.00 & $1.67-5.39$ & $<0.001$ \\
\hline $\operatorname{AFP}(>8.1 \mathrm{ng} / \mathrm{mL}$ vs. $<8.1 \mathrm{ng} / \mathrm{mL})$ & 0.78 & $0.44-1.41$ & 0.418 & - & - & - \\
\hline $\operatorname{NLR}(>2.32$ vs. $<2.32)$ & 2.69 & $1.62-4.46$ & $<0.001$ & - & - & 0.348 \\
\hline Diameter ( $>5 \mathrm{~cm}$ vs. $<5 \mathrm{~cm}$ ) & 2.27 & $1.31-3.95$ & 0.004 & - & - & 0.468 \\
\hline TNM (III+IV vs. I+II) & 2.16 & $1.33-3.51$ & 0.002 & - & - & 0.386 \\
\hline No. of tumors (>1 vs. 1 ) & 1.39 & $1.06-1.82$ & 0.018 & - & - & 0.612 \\
\hline Vascular invasion & 1.14 & $0.69-1.9$ & 0.609 & - & - & - \\
\hline LNM & 2.32 & $1.36-3.96$ & 0.002 & 2.00 & $1.129-3.542$ & 0.017 \\
\hline Surgical margin & 2.40 & $1.29-4.44$ & 0.005 & - & - & 0.121 \\
\hline Differentiation (well/moderate vs. poor) & 1.56 & $0.96-2.53$ & 0.070 & - & - & - \\
\hline MVI & 2.82 & $1.67-4.74$ & $<0.001$ & 2.16 & $1.19-3.92$ & 0.011 \\
\hline LC3B (high vs. low) & 2.33 & $1.41-3.86$ & 0.001 & 2.16 & $1.22-3.84$ & 0.009 \\
\hline
\end{tabular}

OS, overall survival; ICC, intrahepatic cholangiocarcinomas; HR, hazard ratio; CI, confidence interval; $\mathrm{HBV}$, hepatitis $\mathrm{B}$ virus; $\mathrm{CEA}$, carcinoembryonic antigen; CA-125, cancer antigen 125; AFP, $\alpha$-fetoprotein; NLR, neutrophil-to-lymphocyte ratio; LNM, lymph node metastasis; MVI, microvascular invasion; LC3B, microtubule-associated protein 1 light chain 3B.

Table 4. Univariate and multivariate analyses for RFS in ICC patients $(n=105)$

\begin{tabular}{|c|c|c|c|c|c|c|}
\hline \multirow{2}{*}{ Variable } & \multicolumn{3}{|c|}{ Univariate analysis } & \multicolumn{3}{|c|}{ Multivariate analysis } \\
\hline & HR & $95 \% \mathrm{CI}$ & p-value & HR & $95 \% \mathrm{CI}$ & p-value \\
\hline Sex & 1.09 & $0.68-1.75$ & 0.708 & - & - & - \\
\hline Age $(>60$ yr vs. $<60$ yr $)$ & 1.05 & $0.65-1.67$ & 0.853 & - & - & - \\
\hline $\mathrm{HBV}$ & 0.83 & $0.52-1.34$ & 0.449 & - & - & - \\
\hline CEA $(>5.0 \mu \mathrm{g} / \mathrm{L}$ vs. $<5.0 \mu \mathrm{g} / \mathrm{L})$ & 1.78 & $1.00-3.17$ & 0.048 & - & - & 0.860 \\
\hline CA-199 (> $35 \mathrm{U} / \mathrm{mL}$ vs. $<35 \mathrm{U} / \mathrm{mL}$ ) & 1.05 & $0.65-1.71$ & 0.840 & - & - & - \\
\hline CA-125 (> $35 \mathrm{U} / \mathrm{mL}$ vs. $<35 \mathrm{U} / \mathrm{mL}$ ) & 2.91 & $1.63-5.19$ & $<0.001$ & 3.03 & $1.59-5.75$ & 0.001 \\
\hline $\operatorname{AFP}(>8.1 \mathrm{ng} / \mathrm{mL}$ vs. $<8.1 \mathrm{ng} / \mathrm{mL})$ & 0.84 & $0.48-1.47$ & 0.537 & - & - & - \\
\hline $\operatorname{NLR}(>2.32$ vs. $<2.32)$ & 2.83 & $1.75-4.6$ & $<0.001$ & 1.87 & $1.09-3.18$ & 0.022 \\
\hline Diameter (> 5 cm vs. $<5 \mathrm{~cm}$ ) & 2.02 & $1.21-3.37$ & 0.007 & - & - & 0.521 \\
\hline TMN (III+IV vs. I+II) & 2.40 & $1.48-3.89$ & $<0.001$ & - & - & 0.327 \\
\hline No. of tumors (>1 vs. 1 ) & 1.70 & $1.31-2.21$ & $<0.001$ & - & - & 0.064 \\
\hline Vascular invasion & 1.40 & $0.86-2.28$ & 0.173 & - & - & - \\
\hline LNM & 2.66 & $1.53-4.63$ & 0.001 & 2.89 & $1.43-5.86$ & 0.003 \\
\hline Surgical margin & 1.77 & $0.96-3.24$ & 0.066 & - & - & - \\
\hline Differentiation (well/moderate vs. poor) & 1.87 & $1.17-2.98$ & 0.009 & 1.82 & $1.1-3.02$ & 0.019 \\
\hline MVI & 2.88 & $1.7-4.88$ & $<0.001$ & 2.99 & $1.63-5.5$ & $<0.001$ \\
\hline LC3B (high vs. low) & 2.70 & $1.65-4.43$ & $<0.001$ & 3.43 & $1.87-6.29$ & $<0.001$ \\
\hline
\end{tabular}

RFS, relapse-free survival; ICC, intrahepatic cholangiocarcinomas; HR, hazard ratio; CI, confidence interval; HBV, hepatitis B virus; CEA, carcinoembryonic antigen; CA-125, cancer antigen 125; AFP, $\alpha$-fetoprotein; NLR, neutrophil-to-lymphocyte ratio; LNM, lymph node metastasis; MVI, microvascular invasion; LC3B, microtubule-associated protein 1 light chain 3B. 


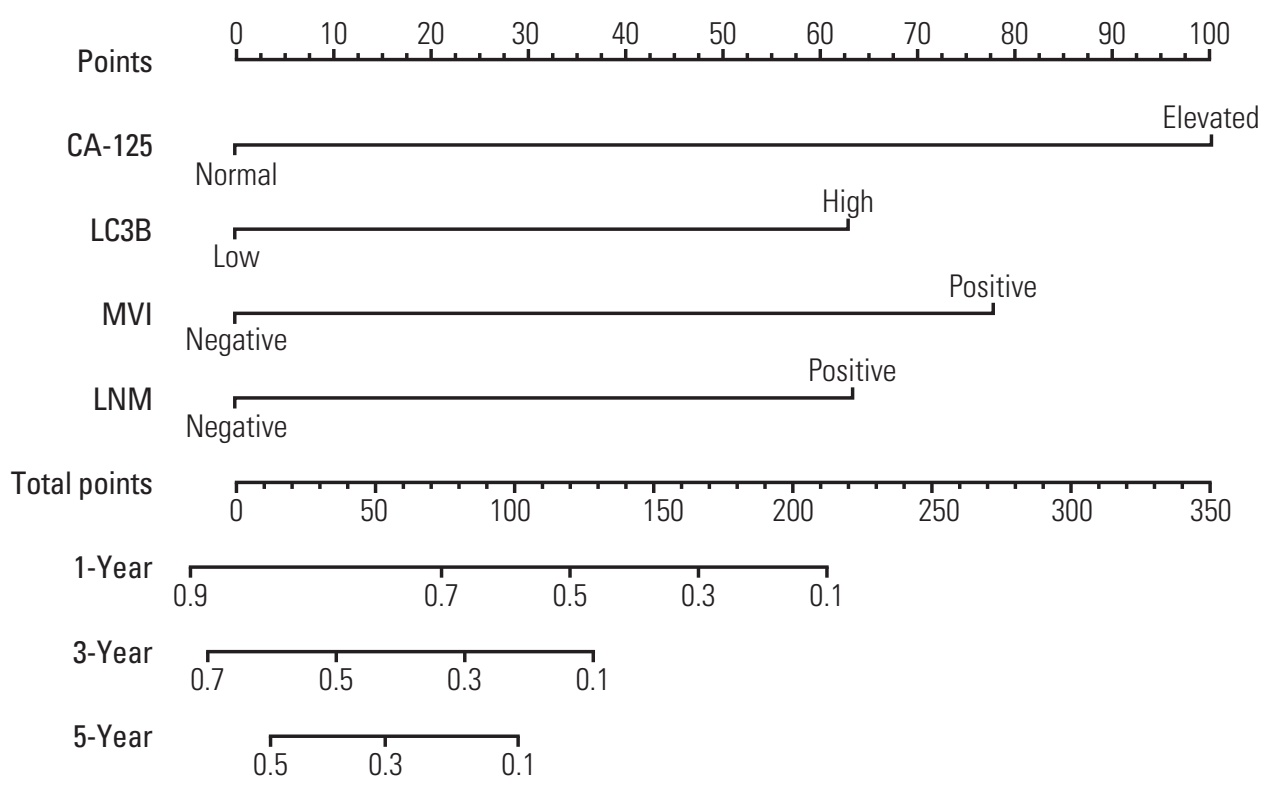

Fig. 2. The nomogram for predicting overall survival of intrahepatic cholangiocarcinoma patients after surgery. CA-125, cancer antigen 125; LC3B, microtubule-associated protein 1 light chain 3B; MVI, microvascular invasion; LNM, lymph node metastasis; OS, overall survival; RFS, relapse-free survival.

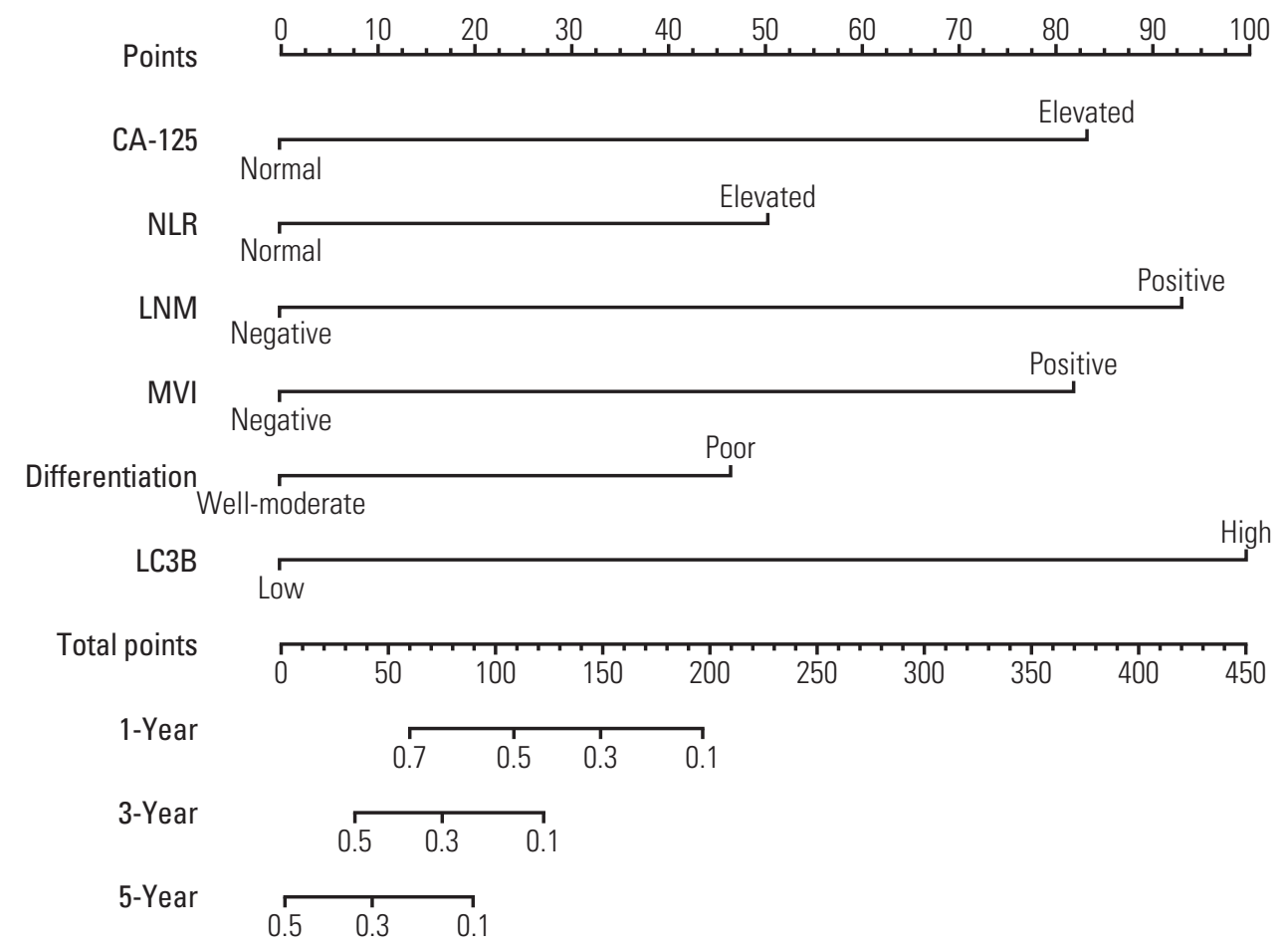

Fig. 3. The nomogram for predicting relapse-free survival of intrahepatic cholangiocarcinoma patients after surgery. CA125, cancer antigen 125; NLR, neutrophil-to-lymphocyte ratio; LNM, lymph node metastasis; MVI, microvascular invasion; LC3B, microtubule-associated protein 1 light chain 3B. 
A

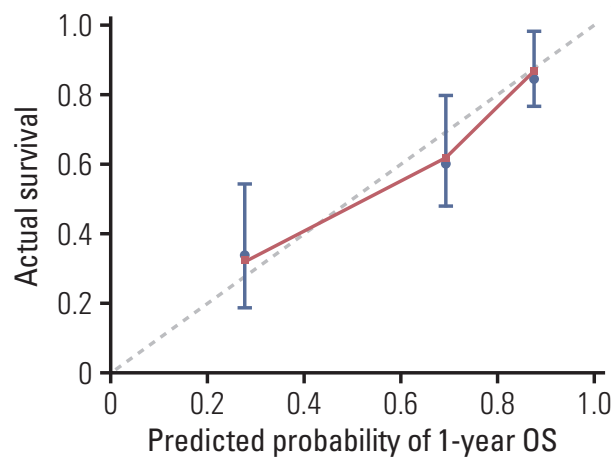

C

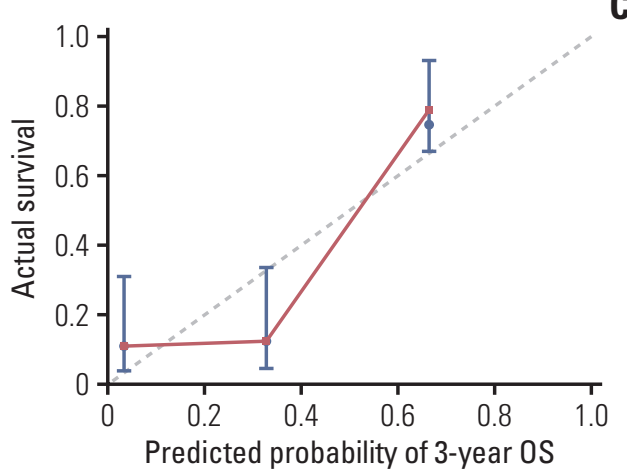

E

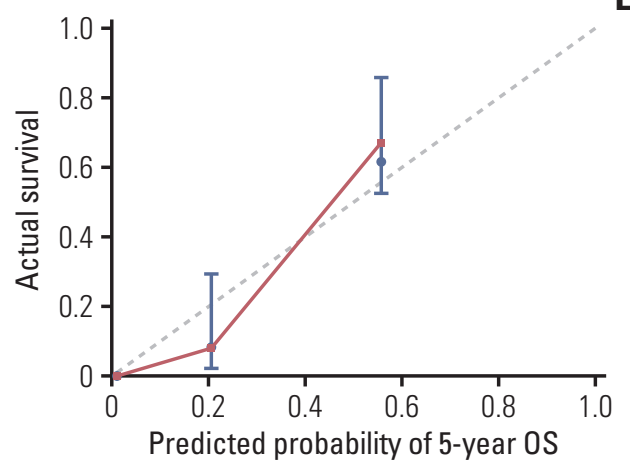

B

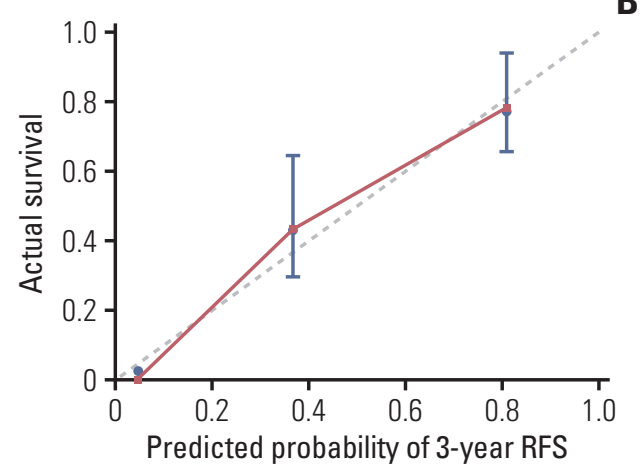

D
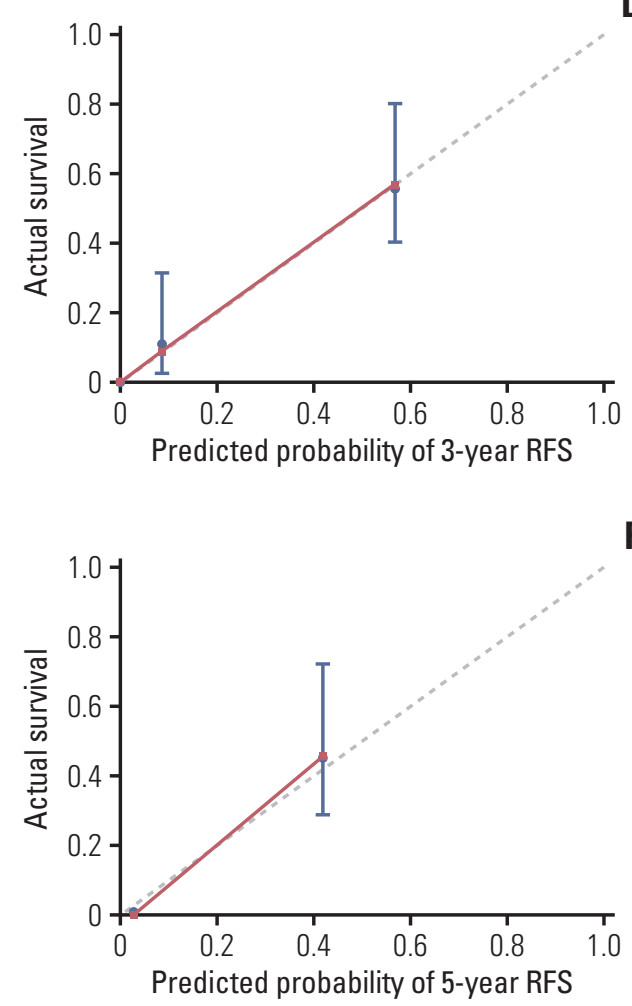

Fig. 4. The calibration curves for predicting patients' postoperative survival. (A) 1-Year overall survival (OS). (B) 1-Year recurrence-free survival (RFS). (C) 3-Year OS. (D) 3-Year RFS. (E) 5-Year OS. (F) 5-Year RFS.

analysis, LC3B expression, CA-125, NLR, LNM, tumor differentiation and MVI were exhibited as significant predictor for RFS. Thus, we also created a nomogram to predict the 1-, 3-, and 5-year RFS integrating the above six independent indicators. To use these nomograms, a vertical line is firstly drawn from the top Point row to the points for each variable. Then, the total points are added up to obtain the 1-, 3-, and 5-year OS and 1-, 3-, and 5-year RFS, respectively (Figs. 2, 3, $\mathrm{S} 1$ and S2 Tables). In addition, the calibration curves for probability of 1-year (Fig. 4A), 3-year (Fig. 4B), 5-year (Fig. 4C)
OS and 1-year (Fig. 4D), 3-year (Fig. 4E), 5-year (Fig. 4F) RFS showed satisfactory accordance between nomogram prediction and actual observation.

\section{Predictive performance of the nomogram in stratifying risk of patients}

To further analyze the discrimination ability of the nomograms, X-tile software was used to determine the optimal cut-off values for total points of each patient gained from the 

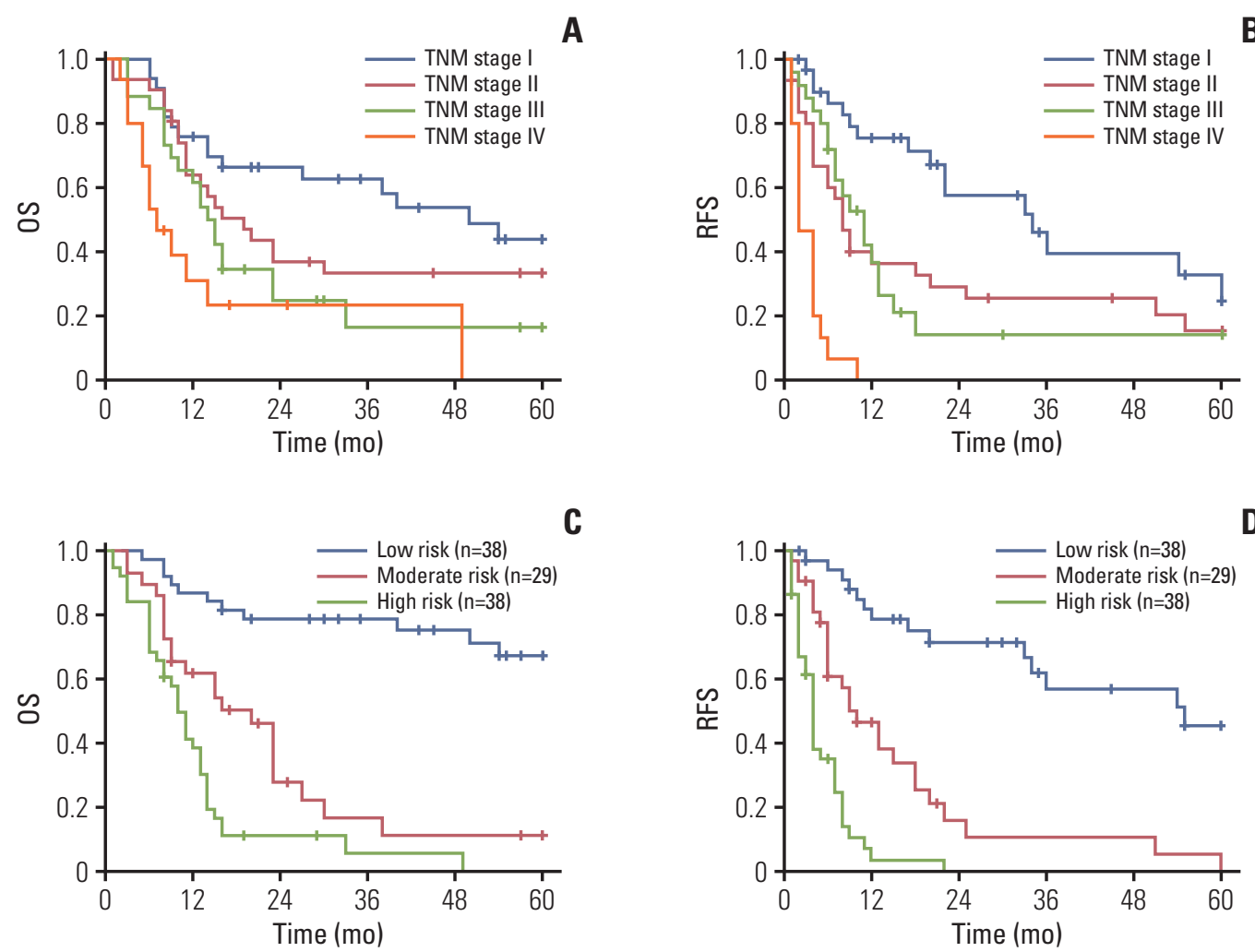

C

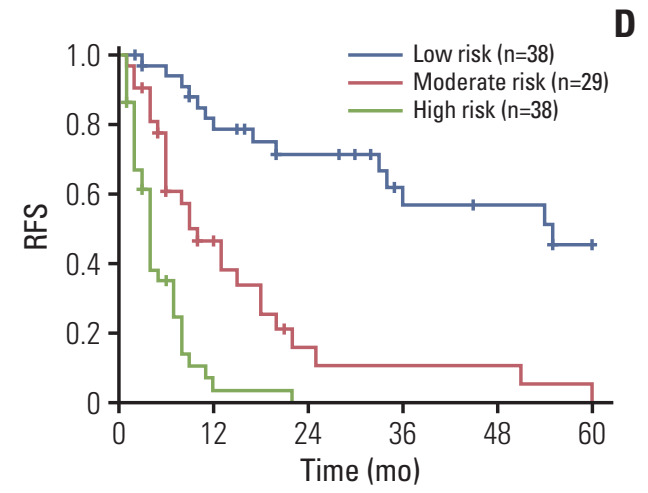

Fig. 5. Kaplan-Meier postoperative survival analysis of overall survival (OS) and relapse-free survival (RFS) according to TNM staging (A, B) and three risk groups stratified by individual total points of nomograms for intrahepatic cholangiocarcinoma patients $(C, D)$ respectively.

A

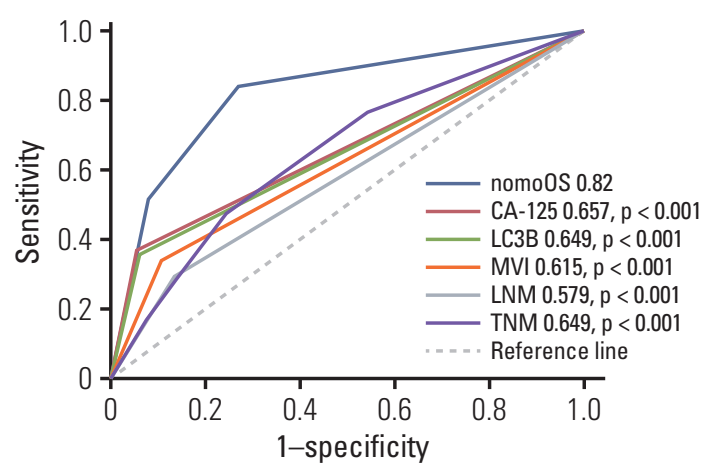

Fig. 6. Sensitivities and specificities for the nomograms and other independent risk factors for postoperative survival of intrahepatic cholangiocarcinoma patients. (A) Area under the curve (AUC) was compared among the nomogram for overall survival (OS), cancer antigen 125 (CA-125), microtubule-associated protein 1 light chain 3B (LC3B), microvascular invasion (MVI), and lymph node metastasis (LNM). (B) AUC was compared among the nomogram for RFS, neutrophil-to-lymphocyte ratio (NLR), LC3B, tumor differentiation, LNM, MVI, and CA-125. 
OS and RFS nomogram. Then the patients were divided into three subgroups (for OS: low risk, 0-62.0; intermediate risk, 62.1-78; and high risk, > 78; for RFS: low risk, 0-82.0; intermediate risk, 82.1-177.0; and high risk, > 177.0) (S3 Fig.). There was significant distinction between Kaplan-Meier curves for OS and RFS risk stratified by the nomogram total points compared with TNM stage (Fig. 5).

\section{Comparison of predictive abilities for OS and RFS bet- ween nomogram and independent risk factors}

Subsequently, we plotted the receiver operating characteristic (ROC) curves for the OS and RFS risk stratified by the nomogram total points, compared to the ROC curves for each independent prognostic factor. The calculated AUC was 0.820 for OS and 0.747 for RFS, which were significantly higher than other independent risk factors $(\mathrm{p}<0.05)$ (Fig. 6). These results suggested that the high discrimination ability of the nomogram for OS and RFS of ICC patients.

\section{Discussion}

It is well known that poor prognostic outcome of ICC patients is attributed to locoregional relapse and/or distant metastasis with limited effective therapies. Autophagy, an evolutionarily catabolic bio-process to degrade intracellular proteins and organelles, was increasingly demonstrating its roles in tumor initiation and development. In this study, we evaluated the correlation between autophagy-related protein expression in ICC and prognostic outcomes.

To our best knowledge, this is the first study to demonstrate LC3B is an independent predictive biomarker for OS and RFS in ICC patients. High-expressed LC3B was significantly associated with poor tumor differentiation, TNM stage, early relapse and unsatisfactory long-term survival. Based on the LC3B expression, we further developed nomograms for stratifying ICC patients and generating therapeutic strategy after hepatectomy.

The role of autophagy in tumor cell-fate decisions is still widely discussed. In the early stage, autophagy mainly played an important role in suppressing tumorigenesis [24], whereas it was also maintained tumor cells survival under stressful conditions, such as hypoxia, anticancer therapy and nutrient deprivation $[12,24]$. In the colorectal cancer researches, inhibition of autophagy by 3-methyladenine or knockdowning autophagy-related protein 7 (Atg7) could significantly strengthen the cytotoxicity of 5-fluorouracil [25]. And Zhao et al. [26] demonstrated the positive effect of autophagy on distant metastasis of prostate cancer. In ICC, suppressed autophagy could enhance tumor chemosensitivity and induce tumor cell apoptosis [27]. Moreover, autophagy is also an inducer of epithelial-mesenchymal transition and increases invasive ability of cholangiocarcinoma cell [28]. In addition, owing to a high risk of relapse and unsatisfactory outcome of ICC patients, increasing studies concentrated the biological features of ICC. However, the relationship between autophagy and the clinical outcome has not yet been explored. In this study, we focused on the association between autophagy and the clinical outcome of patients by IHC staining of the autophagy-related marker LC3B in ICC specimens. LC3B is a specific protein of autophagy, which contains two isoforms. During the autophagic process, the soluble form LC3B (LC3B -I) is lapidated and transforms into the lipidized form LC3B (LC3B-II), which becomes an integral part of membrane of autophagosomes [29]. LC3B is widely used to evaluate the level of intracellular autophagy. Although it was hard to distinguish the isoforms of LC3B by IHC staining, previous studies have indicated that LC3B expression was associated with tumor survival outcome and was a common feature of various types of cancer [30]. Chen et al. [15] enrolled 229 locally advanced breast cancers and performed IHC staining to highlight the expression of LC3B was significantly associated with the biological behavior of anticancer therapy. Liu et al. [31] demonstrated that the high-expressed LC3B in oral squamous cell carcinoma contributed to cancer prognosis and poor survival outcome. El-Mashed et al. [32] showed the clinical significance of LC3B expression in esophageal adenocarcinoma. In the current study, we showed an obvious association between high expression of LC3B and poor clinical outcome (RFS and OS) in patients with ICC after surgery. Multivariable Cox regression analysis indicated that LC3B expression in ICC specimen was an independent predictive marker of RFS and OS. LC3B expression might enable stratification of local relapse/distant metastasis risk in ICC patients with similar clinicopathological features.

In previous studies, different nomograms were established to predict the risk of RFS or OS for patients with ICC after hepatectomy. Yet, most of these studies only focused on the clinicopathological characteristics. To date, these are the first nomograms that established correlation between LC3B expression in ICC tissue by IHC staining and patients' prognostic outcomes. Integration of clinical and laboratory as well as the result from IHC from 115 ICC patients, we found the good fit degrees in these nomograms on predicting RFS and OS. Other than LC3B expression, this nomogram also included tumor differentiation, the presence of LNM and MVI as independent risk factors for reflecting early RFS and OS, which were similar to previously developed stratifying models [20]. Yet, tumor size was not a factor for evaluating the prognostic outcomes in this study, which was controversial for a long time and, even, contained in the AJCC staging sys- 
tems [33]. This could partly explain why TNM staging did not exhibit satisfactory discrimination in survival curves based on different stages. Besides, NLR is incorporated in the nomogram for predicting RFS, which comprehensively reflecting inflammatory status. NLR is commonly identified as an optimal prognostic factor for predicting prognostic outcomes in various types of cancer, which present both directive and indirective roles of neutrophils in tumor development. The increased immunosuppressive activity of neutrophils combined with the reduced cytotoxic activity of lymphocytes is universally considered to engage in tumor progression.

It is worth noting that surgical margin is one of the significant predictive factors in univariate analysis for OS, yet it is not statistically significant in multivariate analysis. While in univariate analysis for RFS, the p-value (0.066) of surgical margin is not significant, though close to 0.05 . The reason could be explained by inadequate sample size of the study. Also, it was possible that the predictive value of surgical margin was undermined due to the confounding effect of other more important prognostic factors.

There are several limitations in the present study. First, we used a retrospective design with recruiting a limited number of patients. Second, we only established stratification systems based on LC3B-related nomogram for evaluating RFS and OS in patients with ICC without an external validation. Furthermore, the nomogram only contained one autophagyrelated marker. Indeed, the whole process of autophagy involves multiple proteins, such as Beclin1, Atg5, and p62. Therefore, a larger sample size clinical study from multicenter is required as external validation of the nomograms.

In conclusion, the present study highlighted the IHC staining of LC3B as an evaluation of autophagy in ICC and demonstrated LC3B as a potential prognostic pattern for predicting OS and RFS in patients with ICC after hepatectomy.
In addition, based on the biological feature of autophagy, we established a nomogram involved LC3B expression, inflammatory index and clinicopathological characteristics, which was considered as a novel stratification system to distinguish ICC patients. It could be applied to concisely predict postoperative survival and assist clinicians to make better individualized treatment recommendations.

\section{Electronic Supplementary Material}

Supplementary materials are available at Cancer Research and Treatment website (https://www.e-crt.org).

\section{Conflicts of Interest}

Conflict of interest relevant to this article was not reported.

\section{Acknowledgments}

This study was supported by Natural Science Foundation of China (81570593, 81770648), Guangdong Natural Science Foundation (2015A030312013), Sci-tech Research Development Program of Guangdong Province (2017A020215023), Sci-tech Research Development Program of Guangzhou City (158100076), Science and Technology Program of Guangzhou City (201508020262, 201400000001-3).

\section{Author Details}

${ }^{1}$ Department of Hepatic Surgery and Liver Transplantation Center, Third Affiliated Hospital of Sun Yat-sen University, Guangzhou, ${ }^{2}$ Guangdong Key Laboratory of Liver Disease Research, Third Affiliated Hospital of Sun Yat-sen University, Guangzhou, ${ }^{3}$ Department of Hepatobiliary Surgery, Affiliated Tumor Hospital of Guangxi Medical University, Nanning, China

\section{References}

1. The general rules for the clinical and pathological study of primary liver cancer. Liver Cancer Study Group of Japan. Jpn J Surg. 1989;19:98-129.

2. Spolverato G, Kim Y, Alexandrescu S, Marques HP, Lamelas J, Aldrighetti L, et al. Management and outcomes of patients with recurrent intrahepatic cholangiocarcinoma following previous curative-intent surgical resection. Ann Surg Oncol. 2016;23:235-43.

3. Zhang H, Yang T, Wu M, Shen F. Intrahepatic cholangiocarcinoma: epidemiology, risk factors, diagnosis and surgical management. Cancer Lett. 2016;379:198-205.
4. Tan JC, Coburn NG, Baxter NN, Kiss A, Law CH. Surgical management of intrahepatic cholangiocarcinoma: a population-based study. Ann Surg Oncol. 2008;15:600-8.

5. Sonbare DJ. Influence of surgical margins on outcome in patients with intrahepatic cholangiocarcinoma: a multicenter study by the AFC-IHCC-2009 Study Group. Ann Surg. 2014; 259:e36.

6. Endo I, Gonen M, Yopp AC, Dalal KM, Zhou Q, Klimstra D, et al. Intrahepatic cholangiocarcinoma: rising frequency, improved survival, and determinants of outcome after resection. Ann Surg. 2008;248:84-96. 
7. de Jong MC, Nathan H, Sotiropoulos GC, Paul A, Alexandrescu S, Marques H, et al. Intrahepatic cholangiocarcinoma: an international multi-institutional analysis of prognostic factors and lymph node assessment. J Clin Oncol. 2011;29:3140-5.

8. Witjes CD, Karim-Kos HE, Visser O, de Vries E, JN IJ, de Man $\mathrm{RA}$, et al. Intrahepatic cholangiocarcinoma in a low endemic area: rising incidence and improved survival. HPB (Oxford). 2012;14:777-81.

9. Levy JM, Towers CG, Thorburn A. Targeting autophagy in cancer. Nat Rev Cancer. 2017;17:528-42.

10. Lai K, Killingsworth MC, Lee CS. The significance of autophagy in colorectal cancer pathogenesis and implications for therapy. J Clin Pathol. 2014;67:854-8.

11. Nah J, Yuan J, Jung YK. Autophagy in neurodegenerative diseases: from mechanism to therapeutic approach. Mol Cells. 2015;38:381-9.

12. Kondo Y, Kanzawa T, Sawaya R, Kondo S. The role of autophagy in cancer development and response to therapy. Nat Rev Cancer. 2005;5:726-34.

13. Lum JJ, Bauer DE, Kong M, Harris MH, Li C, Lindsten T, et al. Growth factor regulation of autophagy and cell survival in the absence of apoptosis. Cell. 2005;120:237-48.

14. Koukourakis MI, Kalamida D, Giatromanolaki A, Zois CE, Sivridis E, Pouliliou S, et al. Autophagosome proteins LC3A, LC3B and LC3C have distinct subcellular distribution kinetics and expression in cancer cell lines. PLoS One. 2015;10: e0137675.

15. Chen S, Jiang YZ, Huang L, Zhou RJ, Yu KD, Liu Y, et al. The residual tumor autophagy marker LC3B serves as a prognostic marker in local advanced breast cancer after neoadjuvant chemotherapy. Clin Cancer Res. 2013;19:6853-62.

16. Wang JY, Wu T, Ma W, Li S, Jing WJ, Ma J, et al. Expression and clinical significance of autophagic protein LC3B and EMT markers in gastric cancer. Cancer Manag Res. 2018;10:1479-86.

17. Cufi S, Vazquez-Martin A, Oliveras-Ferraros C, MartinCastillo B, Vellon L, Menendez JA. Autophagy positively regulates the CD44(+) CD24(-/low) breast cancer stem-like phenotype. Cell Cycle. 2011;10:3871-85.

18. Mikhaylova O, Stratton Y, Hall D, Kellner E, Ehmer B, Drew AF, et al. VHL-regulated MiR-204 suppresses tumor growth through inhibition of LC3B-mediated autophagy in renal clear cell carcinoma. Cancer Cell. 2012;21:532-46.

19. Chen DP, Ning WR, Li XF, Wei Y, Lao XM, Wang JC, et al. Peritumoral monocytes induce cancer cell autophagy to facilitate the progression of human hepatocellular carcinoma. Autophagy. 2018;14:1335-46.

20. Wang Y, Li J, Xia Y, Gong R, Wang K, Yan Z, et al. Prognostic nomogram for intrahepatic cholangiocarcinoma after partial hepatectomy. J Clin Oncol. 2013;31:1188-95.
21. Hyder O, Marques H, Pulitano C, Marsh JW, Alexandrescu S, Bauer TW, et al. A nomogram to predict long-term survival after resection for intrahepatic cholangiocarcinoma: an Eastern and Western experience. JAMA Surg. 2014;149:432-8.

22. Lefort S, Joffre C, Kieffer Y, Givel AM, Bourachot B, Zago G, et al. Inhibition of autophagy as a new means of improving chemotherapy efficiency in high-LC3B triple-negative breast cancers. Autophagy. 2014;10:2122-42.

23. Remmele W, Hildebrand U, Hienz HA, Klein PJ, Vierbuchen M, Behnken LJ, et al. Comparative histological, histochemical, immunohistochemical and biochemical studies on oestrogen receptors, lectin receptors, and Barr bodies in human breast cancer. Virchows Arch A Pathol Anat Histopathol. 1986;409: $127-47$.

24. Ogier-Denis E, Codogno P. Autophagy: a barrier or an adaptive response to cancer. Biochim Biophys Acta. 2003;1603:11328.

25. Lin CI, Whang EE, Donner DB, Du J, Lorch J, He F, et al. Autophagy induction with RAD001 enhances chemosensitivity and radiosensitivity through Met inhibition in papillary thyroid cancer. Mol Cancer Res. 2010;8:1217-26.

26. Zhao R, Bei X, Yang B, Wang X, Jiang C, Shi F, et al. Endothelial cells promote metastasis of prostate cancer by enhancing autophagy. J Exp Clin Cancer Res. 2018;37:221.

27. Hou YJ, Dong LW, Tan YX, Yang GZ, Pan YF, Li Z, et al. Inhibition of active autophagy induces apoptosis and increases chemosensitivity in cholangiocarcinoma. Lab Invest. 2011;91: 1146-57.

28. Nitta T, Sato Y, Ren XS, Harada K, Sasaki M, Hirano S, et al. Autophagy may promote carcinoma cell invasion and correlate with poor prognosis in cholangiocarcinoma. Int J Clin Exp Pathol. 2014;7:4913-21.

29. Mizushima N. Methods for monitoring autophagy. Int J Biochem Cell Biol. 2004;36:2491-502.

30. Sato K, Tsuchihara K, Fujii S, Sugiyama M, Goya T, Atomi Y, et al. Autophagy is activated in colorectal cancer cells and contributes to the tolerance to nutrient deprivation. Cancer Res. 2007;67:9677-84.

31. Liu JL, Chen FF, Lung J, Lo CH, Lee FH, Lu YC, et al. Prognostic significance of p62/SQSTM1 subcellular localization and LC3B in oral squamous cell carcinoma. Br J Cancer. 2014;111:944-54.

32. El-Mashed S, O'Donovan TR, Kay EW, Abdallah AR, Cathcart MC, O'Sullivan J, et al. LC3B globular structures correlate with survival in esophageal adenocarcinoma. BMC Cancer. 2015; 15:582.

33. Edge SB, Compton CC. The American Joint Committee on Cancer: the 7th edition of the AJCC cancer staging manual and the future of TNM. Ann Surg Oncol. 2010;17:1471-4. 